ISSN: $1130-3743$ - eISSN: 2386-5660

DOI: http://dx.doi.org/10.14201/teoredu302247266

\title{
METÁFORAS DE LA EDUCACIÓN UNIVERSITARIA
}

\author{
Metaphors of university education
}

\section{Métaphores de l'éducation universitaire}

\author{
David Luque MENGíBAR* y Ernesto LóPEZ-GÓMEZ*** \\ * Universidad Rey Juan Carlos. Departamento de Ciencias de la Educación, \\ Lenguaje, Cultura y Artes, Ciencias Histórico-Jurídicas y Humanísticas y Lenguas \\ Modernas. C/ Camino del Molino, s/n. 28942 Fuenlabrada, Madrid. \\ david.luque@urjc.es \\ ** Universidad Nacional de Educación a Distancia. Facultad de Educación. \\ Departamento de Didáctica, Organización Escolar y Didácticas Especiales. \\ C/Juan del Rosal, 14.28040 Madrid.elopez@edu.uned.es
}

Fecha de recepción: enero de 2018

Fecha de aceptación: marzo de 2018

\section{RESUMEN}

Este artículo realiza un recorrido por las principales metáforas que se han desarrollado para sintetizar las distintas ideas de la universidad humanista, a saber: la torre de marfil, los grandes libros, la conversación y el agôn. Para ello, desarrolla una aproximación hermenéutica que busca comprender los fines de la universidad, sus instrumentos o la propia institución, a la luz de esas imágenes. Se observa que lo que podría parecer una única interpretación sobre la idea clásica de la universidad es, en realidad, un crisol con diferencias internas muy significativas. El análisis llevado a cabo permite extraer unas notas que permanecen constantes en todas las metáforas, a partir de las cuales es posible repensar la realidad de una universidad de inspiración humanista hoy.

Palabras clave: Universidad; educación universitaria; filosofía educativa; análisis comparativo. 


\section{SUMMARY}

This article presents an analysis of the main metaphors that have been developed to synthesize the different ideas of the humanistic university, namely: the ivory tower, the great books, the conversation and the agon. For this purpose, a hermeneutical approach is developed in order to understand, from these metaphors, the aims of the university, its instruments or the institution itself. After the analysis, it is observed that what could seem a unique interpretation on the classic idea of the university is, in fact, a melting pot with very significant internal differences. The analysis carried out allows us to extract ideas that remain constant in all metaphors, from them it is possible to rethink the reality of a humanist-inspired university today.

Key words: Higher Education; educational philosophy; educational theory; comparative analysis.

\section{SOMMAIRE}

Cet article présente une analyse des principales métaphores qui ont été développées pour synthétiser les différentes idées de l'université humaniste, à savoir: la tour d'ivoire, les grands livres, la conversation et l'agon. A cet effet, une approche herméneutique est développée afin de comprendre, à partir de ces métaphores, les objectifs de l'université, ses instruments ou l'institution elle-même. Après l'analyse, on observe que ce qui peut sembler une interprétation unique de l'idée classique de l'université est, en fait, un melting-pot avec des différences internes très significatives. Cette analyse nous permet d'extraire des idées qui restent constantes dans toutes les métaphores, étant d'eux il est possible de repenser aujourd'hui la réalité d'une université d'inspiration humaniste.

Mots clés: Université; l'enseignement supérieur; métaphores; philosophie de l'éducation.

\section{INTRODUCCIÓN}

Que el recurso de la metáfora juega un papel fundamental en el conocimiento humano es algo que ya hoy día parece demostrado (Searle, 1993; Glucksberg y Keysar, 1993; Lakoff y Johnson, 1980). En el ámbito pedagógico, el uso de la metáfora también se ha dado en referencia al aprendizaje (Petrie y Oshlag, 1993), al pensamiento del profesorado (Martínez, Sauleda y Huber, 2001), a la intervención socioeducativa (Úcar, 2018) y, especialmente, a la teoría de la educación (Amilburu, 1997). De este modo, las metáforas han ejercido una importante función epistemológica porque permiten recrear mentalmente ideas complejas a partir de conceptos en apariencia alejados y relativamente más sencillos de comprender.

Pero, en lo que tiene que ver con el ámbito exclusivo de la filosofía educativa (FE, en adelante), han nacido nuevos centros de interés que han reclamado su 
atención de un modo específico. Quizá uno de los más señalados es la educación superior. En efecto, como señaló el profesor Curren $(2007,2017)$, la FE no se ha prodigado en una reflexión específica sobre la idea de universidad hasta muy recientemente. La expresión liberal education había servido para integrar todas las interpretaciones de una idea clásica de universidad, pero la complejidad actual de los sistemas universitarios ha hecho necesaria una nueva mirada. Y es en esa intersección entre el uso de las metáforas en la teoría educativa y los nuevos ámbitos de reflexión de la FE que comienza a cobrar sentido este artículo. Su fin es sintetizar las principales metáforas de la educación universitaria que articulan diversas teorías educativas, buscando comprender los fines de la universidad, sus instrumentos o la propia institución a la luz de esas imágenes. Pero, antes de proceder al análisis, parece pertinente preguntarse si podrían existir otros motivos que justifiquen este texto, en un ambiente como el actual, donde prima el interés por lo práctico en la reflexión educativa.

El primer elemento casi nace de una continuación de la reflexión epistemológica anterior. Que después de la crisis analítica de la FE llegaron nuevos investigadores al área, con nuevas sensibilidades, que comenzaron a centrar su interés en elementos que habían pasado desapercibidos a la generación anterior. Y eso en nuevas comprensiones de la propia FE que defendían que el objeto de la propia disciplina residía en una comprensión filosófica, sin que ello significara preocuparse necesariamente de mejorar la práctica educativa -al uso de lo que sucedería en la filosofía de la ciencia (Siegel, 1981, 127)-. Desde estas premisas, el mero hecho de querer analizar y comprender esas metáforas en un contexto universitario como el actual, tan ajeno y lejano a lo que era la propia universidad hace veinte años, justificaría este artículo.

Pero, además del argumentario epistemológico previo, lo cierto es que han proliferado varios textos que han defendido la idea de una universidad humanista frente a su mercantilización. Vistos desde lejos, todos esos escritos parecían estar inspirados en una visión compartida, unificada y sólida de lo que debería ser una universidad inspirada en ideales humanistas. Pero, siendo esto cierto en algún sentido, la realidad es que las diferencias son sustanciales cuando se analizan de cerca. Baste leer, por citar solo a algunos, a Javier Gomá (2016), Miguel GarcíaBaró (2014), Víctor Pérez-Díaz (2010) o Jordi Llovet (2011) para corroborar esto. Y en base a esta discrepancia de interpretaciones, parece necesario un intento de sistematización de esos elementos a priori dispersos.

Además, y esto ya en lo que tiene que ver con la identidad de los académicos (López-Gómez, 2015; Martínez, Sauleda y Huber, 2001), algunos estudios concluyen que uno de los factores que contribuyen a su desarrollo es la representación que tienen sobre la idea de universidad y el papel que esta desempeña en sus proyectos de vida. En palabras de Clegg (2008, 339), «universities and academia are imaginary spaces as well as lived and experienced ones. In the negotiation of identity these imaginaries are important». Esta misma idea se refuerza cuando lo que se propone a los académicos son metáforas que sinteticen esas imágenes 
(Visser-Wijnveen et al., 2009, 675-676). Ahí, las metáforas de la educación superior "help people to express thoughts which are difficult to express otherwise» (VisserWijnveen et al., 2009, 675). Y más todavía: "The metaphor can be used as a vehicle to say what is actually meant but is difficult to explain" (Visser-Wijnveen et al., 2009, 675). Así que, en suma, un estudio sobre las metáforas de la educación superior puede contribuir a ayudar a los profesores a comprender la institución en la que trabajan y el tipo de académicos que desean ser.

En definitiva, este artículo trata las principales metáforas de la educación universitaria que pueden arrojar luz sobre las diversas interpretaciones de la universidad desde un punto de vista humanista. Su orden de análisis sigue un progreso lógico que atiende, más que a la cronología, a la presencia de matices que permiten una mayor profundización en la metáfora siguiente, con una excepción: la primera metáfora será, justamente, la que ha venido significando la idea clásica de universidad, al abarcar un arco temporal de milenios. Todas las demás, ya sí, aparecerán según esa progresión lógica descrita.

\section{PRIMERA METÁFORA. LA UNIVERSIDAD COMO TORRE DE MARFIL}

La metáfora de la torre de marfil (ivory tower) se usa con cierta asiduidad incluso hoy, si bien sus raíces y FE son bastante desconocidas. Presentamos, en primer lugar, una breve síntesis sobre el nacimiento de la metáfora y cómo llega a la idea de universidad y, en segundo lugar, los elementos esenciales para comprender la fE que se encierra en ella.

\subsection{Raíces bistóricas de la "torre de marfil» y trasposición a la idea de universidad}

El nacimiento de la imagen no tiene que ver con un contexto estrictamente universitario ni con ese sesgo negativo actual. Su origen se halla en la antigüedad, más concretamente en el mundo bíblico y el devocionario mariológico, que le otorga las resonancias de pureza, así como en el canto XIX de la Odisea, que le otorga el matiz de la separación por cuanto Penélope anhela que Odiseo regrese y lo imagina cruzando las puertas de marfil de la habitación donde teje (Shin, 2018, 37; Shapin, 2012, 2-4). Poco a poco, la idea de la torre de marfil comienza a aplicarse a la universidad y es ahí que la idea de pureza y aislamiento adquiere esos ecos negativos. Este proceso culmina, sobre todo, en el siglo XIX y la llegada de las ideologías totalitarias a Europa en el xx (Shin, 2018, 37-38; Shapin, 2012, 6-10). Entonces, muchos intelectuales tomaron partido por alguna de las facciones en contienda y se reservó la idea de la torre de marfil para quienes pensaron que su función como académicos era el puro desvelamiento de la verdad. La Guerra Fría y sus políticas de investigaciones aplicadas no hicieron sino contribuir a que se asentara esta idea, que ha ido evolucionando en tensión entre un mundo escolástico que todavía conserva algunos reductos y un mundo aplicado que impregna 
la misma idea de universidad moderna (Shapin, 2012, 16-17; Etzkowitz et al., 2000, 326). ¿Pero dónde se encontraría la última gran defensa de esta idea justo antes de la gran transformación de la universidad, del asentamiento definitivo de la «torre de marfil» como algo negativo?

\subsection{John Henry Newman: bermenéutica de dos fragmentos}

Parece evidente que ese proceso de mercantilización que se comentó al comienzo del artículo ha provocado que casi todas las disciplinas que componen la universidad actual miren hacia la sociedad, el mercado o el ejército como el destino natural de sus investigaciones (Etzkowitz et al., 2000, 328-329). Acaso la excepción a este respecto sean las facultades de teología y, más concretamente, la comprensión de la teología que se deduce de la teoría educativa de John Henry Newman, quien es considerado como el último gran representante y apologeta de la ivory tower (Chantler, 2016, 217-218). Así lo piensan tanto algunos autores, que le marcan como un hito en la historia de la universidad católica, como incluso el propio magisterio de la Iglesia (MacIntyre, 2009, 2012; Fuller, 2003, 37; Ex Corde Ecclesiae, nn. 4, 16, 23; Veritatis Gaudium, n. 4). Así que, en suma, parece necesario aproximarse a Newman, puesto que hay una hermenéutica posible de su pensamiento que condensa la metáfora de la «torre de marfil» en la correlación de dos de sus ideas. Por una parte, que el conocimiento es un fin en sí mismo (Newman, 2011, 143-144; Bottone, 2010; Tingley, 2002). Por otra parte, que la universidad es una comunidad de profesores (Newman, 2011, 125).

La afirmación de que el conocimiento es un fin en sí mismo tomó una primera dirección apologética que desarrollaba una $\mathrm{FE}$ en contraposición al utilitarismo propuesto por John Locke, que se refinó en sus seguidores de la Edinburgh Review (Bottone, 2010, 104, 116-117; Culler, 1958, 220-226; McGrath, 1951, 134136; Shrimpton, 2014, 74). Frente a ellos, quienes sostenían que la universidad debía facilitar la incorporación de los estudiantes al mercado de trabajo, Newman defendió la necesidad de adquirir una mente ordenada sin ningún tipo de fin más. Adquirir, dicho de otro modo, un sistema mental de todos los conocimientos que contribuían a una comprensión de la realidad, de las relaciones entre ellos, de sus límites (Newman, 2011, 32-38, 102-104, 133-135, 140-142). La otra dirección condujo a Newman a rechazar cualquier otra dimensión teleológica, llegando a negar incluso la formación moral o religiosa de los estudiantes (Newman, 2011, 140-142). Lo que hizo Newman para llegar a este argumento fue una trasposición radical de la teoría aristotélica de las virtudes a la organización institucional de la universidad (Hochschild, 2003). El resultado era una universidad que aparecía como dividida en dos instituciones. La universidad en sí misma, que sería el edificio donde se adquirían las virtudes intelectuales que he descrito anteriormente: donde los profesores enseñaban un conocimiento teórico a los estudiantes (Newman, 2011, 30-31 125). Y el college, que es el lugar donde los estudiantes vivían con un tutor y perseguían las virtudes morales (Bottone, 2010, 69-71; Shrimpton, 
2014, 476). Hay que señalar que Newman fue consciente del punto hasta el que había extremado sus argumentos ya al final de sus discursos. Entonces, lo matizó con la famosa descripción del caballero, con la intención de poner en evidencia qué sucedería si no se modulaba la formación del intelecto con la religión (Wright, 1999, 239), y con el cierre que refiere a san Felipe Neri (Tillman, 2008). Pero estas modulaciones no sirvieron para evitar que la universidad que proponía Newman se viera como una apología clasista (Martin, 1994, 70-87, 170-186). Antes bien, como indicamos más arriba, se reforzaron en la correlación con la descripción que hizo de lo que debería ser una universidad ya casi sociológicamente, donde todo estaba impregnado por una trasposición literal del intelectualismo de las premisas precedentes: un «conjunto de hombres sabios, celosos por sus respectivas ciencias, y mutuamente rivales, que se ven llevados, por trato familiar y en favor de la paz intelectual, a armonizar sus pretensiones y relaciones de disciplinas» (Newman, 2011, 125) en lo que se refería a los profesores, y el único fin de adquirir un "hábito de la mente que dura toda la vida, y cuyas características son libertad, sentido de la justicia, serenidad, moderación y sabiduría. [...] en suma lo que en un discurso anterior me he atrevido a denominar hábito filosófico» (Newman, 2011, 125), en lo que se refiere a los estudiantes.

Así, en suma, la correlación de estos dos argumentarios contribuye a representar la torre de marfil en tanto aísla la comunidad de profesores, estudiantes y usos del conocimiento de la sociedad. Por otra parte, salvo una breve crítica al escolasticismo, Newman no se refiere al uso o a la ausencia de libros en los métodos de enseñanza-aprendizaje. La abstracción de su discurso - pese a la inclusión de una segunda parte en el proyecto primero de la Idea- le obligó a dejar de lado estas consideraciones que, sin embargo, tomarán fuerza en una relectura posterior de la idea clásica de universidad que constituirá una metáfora en sí misma. La de los Great Books.

\section{SEGUNDA METÁFORA: LOS GRANDES LIBROS DE LA HISTORIA}

Robert M. Hutchins, que citó a Newman en The Higher Learning in America (Hutchins, 1936, 63, 103) y La universidad de Utopía (Hutchins, 1959, 16-17), protagonizó un gran debate público con John Dewey sobre sus respectivas formas de comprender la educación (Heldke, 2005; Scott Johnston, 2011; Bruce, 2011; Jover y Gozálvez, 2012, 40-43). Precisamente en esta discusión, Hutchins usó la expresión «Ivory Tower». Primero lo hizo para rechazar las críticas a su teoría, que desarrollaremos a continuación (Shapin, 2012, 47). Más tarde, cuando EE. UU. tomó parte en la Segunda Guerra Mundial, para reconocer que los tiempos de la torre de marfil habían terminado debido a la urgencia de ganar la guerra (cit. en Shapin, 2012, 16). Con todo, lo cierto es que usó la expresión que más en boga estaba en la época, lo que significa que no pensaba realmente que esa imagen condensara su teoría educativa. De hecho, la concreción que adquirió su idea en colaboración con Mortimer J. Adler siguió unos derroteros que le otorgan identidad propia. Más 
que hablar de una «torre de marfil» habría que hablar de una colección de libros. De una exigua biblioteca de más o menos cien ejemplares de obras clásicas. De great books (GB en adelante). Para ello, como en el caso anterior, articularemos dos apartados: una breve síntesis histórica sobre la génesis de la idea y una exposición de la FE que deriva de ella.

\subsection{Génesis de los Great Books: del victorianismo a Mortimer J. Adler}

La idea de los GB no nació con Hutchins y Adler, sino en la Inglaterra victoriana de Newman (Chaddock y Cooke, 2015, 204-209). Al filo del XIX, comenzó a asociarse a una lista de libros que recogía lo mejor que ha sido dicho o pensado. Pero es en el victorianismo norteamericano donde esta idea se introdujo en la universidad (Lacy, 2006, 22-84; Lacy, 2008, 413; Lacy, 2010, 118-119; Lacy, 2013, 1-3). A pesar de algunos antecedentes, Columbia University fue la institución que más contribuyó a adoptar esta perspectiva (Lacy, 2008, 425-433; Lacy, 2010, 121-122; O' Banion, 2016, 328-330). Implantaron lo que se llamó core curriculum, a saber, unos programas que se ofertaban a estudiantes de todas las disciplinas. Entre ellos se encontraba el que llevaba a cabo un profesor llamado John Erskine, basado en la lectura de un canon de libros. Allí, entre los estudiantes que se sintieron atraídos por esta propuesta, estaba un jovencísimo Adler, quien, a pesar de haber estudiado ya con Dewey (Adler, 1977a, 29), se impresionó tanto por la FE que encerraba la propuesta de Erskine (Adler, 1977a, 15-35) que, cuando Hutchins lo llamó para trabajar junto a él en la Universidad de Chicago que presidía, ambos se convirtieron en los grandes defensores de una democracia cultural (Dzuback, 1990).

\subsection{La filosofía educativa de los Great Books: The paideia movement}

Para los anales de la historia, Hutchins ha quedado vinculado a la teoría educativa conocida como General Education mientras que Adler aparece como el artífice de Paideia Proposal (Johnson, 1985; Stevens, 2001; Heldke, 2005; Lacy, 2006, 2008, 2010). La realidad es que ambos participan de una actualización de la educación liberal a través de una relectura de los GB. ¿Pero qué son los GB? "A classic is a book that is contemporary in every age" (Hutchins, 1936, 78). El propio Hutchins, ya desde un punto de vista pedagógico, se preguntó «can we call a man educated who has never read any if the great books in the western world?» (Hutchins, 1936, 78). En efecto, esta FE sostenía que no se podía hablar de una educación propiamente dicha si los estudiantes no habían leído los GB confrontándose directamente con el texto del autor. Ahora bien, la razón para sostener este argumento no era un clasicismo trasnochado, sino que esos libros ayudaban a comprender cualquier disciplina o problema contemporáneo en tanto "cover every department of knowledge» (Hutchins, 1936, 81). Pero la novedad radical de esta FE residió en que adaptaron su teoría a un currículum nacional que cubría 
todos los tramos de edad, también si alguien no podía acudir a la universidad (Adler, 1988, 210-224).

Este programa consistía en tres etapas integradas (Adler, 1977, 227; Adler, 1982, 31-32, 51; Adler, 1988, 285-288, 239-308). En la primera etapa se haría necesaria la adquisición de un conocimiento organizado a través de libros escolares y su debida instrucción. En la segunda, ya con los instrumentos cognitivos básicos adquiridos, se profundizaría en el desarrollo de destrezas intelectuales que capacitaban para una lectura profunda de los textos, así como para la escritura, la oratoria y la escucha. Y una última etapa que se extendería a la adquisición personal de ideas, cuyo proceso era un diálogo abierto entre los participantes a través de un ejercicio mayéutico a la luz del estudio de los GB.

Pero esta teoría, como ya sugerimos, tenía profundas dimensiones políticas. Adler hacia reposar el fundamento de su teoría política en una correlación sociológica propia de los Estados modernos. Que sufragio universal y escolarización universal son una misma cosa (Adler, 1982, 3). De esa premisa, como decíamos, Adler deducía consecuencias teleológicas que han de comprenderse políticamente: "That a democratic society must provide equal educational opportunity not only by giving to all its children the same quantity of public education -the same number of years in school- but also by making sure to give to all of them, all with no exceptions, the same quality of education» (1982, 4). Y así, lo que a ojos de muchos era una postura conservadora y elitista (Stevens, 2001, 171-183; Lee, 2012, 4-6, 23-25) se volvía progresista por socialista (Weltman, 2002; Lacy, 2010). Porque buscaba hacer realidad una democracia cultural a través de su Paideia Proposal: extender la alta cultura a todas las capas de la sociedad para elevar el nivel intelectual, moral, espiritual y cívico de la nación (Adler, 1982, 16-17). Algo que, en el pensamiento adleriano, unía a los que él denominó "great american educators": Dewey y Hutchins (Adler, 1982, 3-6). A esta democratización de la alta cultura que se consigue a través de la educación hay que añadir, todavía, tres elementos fundamentales (Adler, 1982). El primero, que ha de erigirse como un objetivo común a todos los estudiantes y mantenerse en todos los niveles, en mayor o menor grado. El segundo, que ha de ser lo bastante dúctil como para adaptarse a las diferencias personales, culturales e históricas. Esto pasaría por considerar las escuelas como comunidades cuya peculiaridad es que los ciudadanos que conformaban esas comunidades eran profesores y estudiantes unidos por su deseo de aprender. El último, que se necesita considerar la educación como un aprendizaje para toda la vida (Adler, 1988, 81-90). Y esto significa que es necesario sustentar su teoría educativa en unas coordenadas antropológicas básicas, a saber, que el hombre tiende a conocer y esto da sentido al lifelong learning (Adler, 1982, 9-12; Adler, 1988, 210-224) y que el conocimiento se relaciona con la felicidad con independencia del trabajo que se desempeñe (Adler, 1982, 77-84).

En definitiva, Hutchins y Adler tomaron una tradición que les precedía y la recondujeron en un movimiento que miraba al pasado, donde los libros emergían como el punto de unión con todas las ideas precedentes que habían configurado 
la humanidad, y un movimiento hacia el futuro, en esa interpretación política de una democracia cultural que habría de exceder los estrechos límites de la educación formal. Pero su propuesta, a pesar del enorme éxito editorial que supuso, no pareció arraigar en el seno interno de la FE más especializada, que prácticamente nacía en la misma época. Más que la metáfora del libro -del clásico, si se prefierese desarrolló la metáfora de la conversación.

\section{TERCERA METÁFORA: LA CONVERSACIÓN}

Al hilo del sueño de la democracia cultural apareció lo que Hutchins llamó "Centros de pensamiento". Lugares donde todos los adultos acudirían a conversar sobre los GB una vez habían finalizado su época de estudios, llegaran estos al nivel que llegasen. Pero lo cierto es que, visto el papel de la conversación en el conjunto de su filosofía educativa, parece más un elemento que responde a necesidades retóricas o excesivamente prácticas que a una de las deducciones principales de sus premisas. No sucede así, con Michael Oakeshott y la metáfora que ha dejado para la educación universitaria: la idea de universidad como conversación.

\subsection{Michael Oakeshoot y la filosofía de la educación: algunas precisiones previas}

Oakeshott llegó a la FE debido a Richard Stanley Peters, aunque él siempre se dedicó a la filosofía política en la London School of Economics. La influencia de su pensamiento educativo, que excede su participación en The Concept of Education (Peters, 1968), se aprecia en filósofos de la educación posteriores, como Maxine Green o René Arcilla (Williams, 2007; Williams, 2010). Hay que precisar algunos elementos sobre la relación entre Oakeshott y la educación. Que, aunque procede del mundo inglés y defiende una idea de educación liberal y pese a que se puede pensar que conoce el pensamiento newmaniano (Oakeshott, 2009b, 50; 2013, 132) e incluso comparte algunos criterios con él (Fuller, 2003-2004, 39), no parece que sea así en la teleología que maneja (Oakeshott, 2009b, 94; Standish, 2003, 222-223; McIntyre, 2013, 343-344). Que además, y aunque Oakeshott concede una importancia fundamental al papel de los clásicos en una educación política, no parece que esté de acuerdo con la interpretación de Hutchins y Adler (Williams, 2010, 226-228; Fuller, 2009, 10). Y que Oakeshott ha sido situado dentro del marco más amplio de la metáfora de la educación como iniciación (Amilburu, 1997, 130). Hay que señalar que esto es acertado, muy congruente, porque en Oakeshott conviven esas dos metáforas. Cuando se refiere a la educación in genere, ahí, sugiere que es una iniciación en una herencia cultural. Ahora bien, cuando su pensamiento se centra en la universidad, entonces, la metáfora se transforma en la conversación. Williams lo explica cuando sostiene que esta última «is Oakeshott's central organising metaphor and it refers to the special conversation constituted by participation in a cultural inheritance into which education offers an initiation» (Williams, 2010, 
224). Así, la conversación es «a metaphor recurring throughout Oakeshott's writing on humanity, freedom and education» (Engel, 2007, 332). Pero, si son así las cosas, ¿qué es la conversación en el pensamiento oakeshottiano y qué implicaciones tiene para una teoría de la educación? Antes de llegar a la misma metáfora nos parece necesario comprender cómo se construye su teoría educativa desde la relación con algunos de los elementos más relevantes de su filosofía política.

\subsection{La idea de universidad como conversación}

Se puede decir que el escepticismo de Oakeshott le hizo un hombre conservador. Había comprendido que todo el pensamiento político de su época se encontraba inmerso en un contexto profundamente racionalista, donde el sometimiento de la realidad a la razón positivista se erigía como la única forma de política posible. No se trataba de una cuestión de izquierdas o derechas, sino de una manera reductiva de comprender la realidad que, ya traducida a una moral y una legislación, imponía un único modo de vida a todos los miembros de una comunidad. O, dicho de otra manera, la política quedaba reducida a mera técnica (Oakeshott, 2002; Oakeshott, 2017, 42-51; Franco, 2004, 81-115). Frente a esto, Oakeshott articuló una perspectiva alternativa. Rechazaba, como decíamos, que exista un único modo de vida, pero no que existan comunidades cuya moral y cuyas leyes puedan ser válidas para el desarrollo de una vida desde una perspectiva local. De aquí nacerán las distinciones que Oakeshott realizó a lo largo de su obra: «asociación qua empresa» y "asociación civil» (Oakeshott, 2003; 2013, 119-158; Frowe, 2007, 272-275), "estilo ideológico» y "actitud conservadora» (Oakeshott, 2017) o "política de fe» y "política de escepticismo» (Oakeshott, 2017; Frowe, 2007, 265-272).

Ahora bien, las comunidades que nacen como asociación civil o con actitud conservadora o desde la política del escepticismo se configuran a partir de una comprensión particular de la "tradición». Esta no puede pensarse como un constructo rígido (Himmelfarb, 1975). Antes bien, Oakeshott le otorga a la tradición una propiedad interna maleable y se adapta a la situación en base al conocimiento acumulado (Oakeshott, 2009a, 51, 74-75). En sus propias palabras, en «el corazón de toda forma de vida tradicional existe espacio para la libertad y la creatividad, y la desviación puede ser una expresión de esa libertad, que surge de una sensibilidad hacia la tradición misma de permanecer fiel a la forma tradicional» (Oakeshott, 2017, 99). Esta es, por cierto, una de las razones por las que el pensamiento del filósofo inglés pervive en muchos filósofos de la educación actuales. Así que, por ello, parece lógico preguntarse por el papel de la educación que nace de esta filosofía política.

La FE de Oakeshott nace de dos consideraciones. Que el hombre es un ser inacabado y que las comunidades políticas están formadas por una herencia de pensamientos humanos, expresados en lenguajes tan diversos como la música, el arte, la matemática o la filosofía, que pueden ser comprendidos por cuanto son expresiones de seres humanos brillantes (Oakeshott, 2009b, 39-40, 69). Y ahí, en 
la intersección entre una concepción y otra, nace la educación como una iniciación en la que los estudiantes son introducidos en una cultura sedimentada por el tiempo a través de la que conocen el mundo y se comprenden a sí mismos (Amilburu, 1997; Tripp, 1992, 3-5; Engel, 2007, 339): «Porque la única manera de llegar a ser humano es entrando en él [legado], y habitar en él equivale a ser un ser humano" (Oakeshott, 2009b, 70). Y es aquí, en estos procesos de adquisición, donde emerge la metáfora de la conversación:

Tal vez podamos pensar en estos componentes de la cultura como voces, y en cada una como la expresión de una interpretación bien definida y condicional del mundo y un idioma bien definido de autocomprensión humana, y en la cultura en sí como estas voces unidas, de la única forma en que podrían juntarse estas voces, en una conversación; una aventura intelectual improvisada e infinita, en la que imaginariamente entramos en una variedad de modos de comprender el mundo y a nosotros mismos y no nos desconciertan las diferencias ni nos dejamos abatir por el carácter inconcluso de todo. Y tal vez podemos reconocer el aprendizaje liberal, sobre todas las cosas, como una educación en la imaginación, una iniciación en el arte de esta conversación en la que aprendemos a reconocer las voces; a distinguir los diferentes modos de enunciados que tienen, a adquirir los hábitos intelectuales y morales apropiados para esta relación conversacional y, de ese modo, a hacer nuestro debut dans la vie humaine (Oakeshott, 2009b, 62).

Para Oakeshott, todas las experiencias de conocimiento son expresiones del pensamiento del ser humano que se esfuerzan por conversar entre sí, sin ningún tipo de superioridad entre ellas (Oakeshott, 2009b, 75; McIntyre, 2013, 356). Ya sugerimos que no puede haber un único fin para todos porque no puede haber ningún proyecto político que imponga un modo de vida universal y porque la tradición tiene un componente maleable y creativo. El corolario pedagógico de esta idea es que aprender es conocer esas voces que componen la cultura y conocerse a sí mismo a través de ellas para encarnar una historia propia (Oakeshott, 2009b, 62, 90; McIntyre, 2013, 355; Engel, 2007, 340-341). Y, por esto mismo, cada estudiante tiene "freedom to pursue her own aims with minimal frustration" (Engel, 2007, 341). Pero en lo que tiene que ver con los elementos más específicos de la universidad, la metáfora de la conversación le proporciona algunos atributos fundamentales (Engel, 2007, 341; Fuller, 2003-2004, 43).

Oakeshott se suma a los autores precedentes en tanto que la educación supone una actividad en sí misma o libera al hombre de cualquier otra actividad práctica, subrayando la vinculación entre el ocio y el estudio (Gary, 2017, 85; Gary, 2006). Se podría decir que "more than the foundation of freedom, liberal education is also the expression of freedom» (Engel, 2007, 340). Esta idea lleva a Oakeshott a rechazar cualquier mezcla de la universidad con la sociedad, pues no debe proporcionar un conocimiento técnico que ayude a los estudiantes a encontrar mejores puestos de trabajo (Oakeshott, 2009b, 143-144; Fuller, 2003-2004, 47). Los estudiantes acuden a la universidad «en busca de su destino intelectual» (Oakeshott, 2009b, 139). Y los profesores ya no tienen solo la tarea de enseñar, sino que deben, además, buscar 
el conocimiento (Oakeshott, 2009b, 136; Fuller, 2003-2004, 40). Ahí, en la relación íntima que se da entre el académico y la disciplina que cultiva, emerge una voz. La voz no es solo la del académico, que quedaría reducida al contexto específico de la universidad, sino mucho más ampliamente la de cada disciplina. "Each language has its own logic of explanation, which has emerged historically from within the practice of the lenguage itself» (McIntyre, 2013, 356). Claro que la cantidad de académicos y la fragmentación de las disciplinas puede dar la idea de escasa unidad. Pero, siendo esto cierto, en el pensamiento de Oakeshott la solución no estriba en una disciplina más encargada de unificar las conclusiones de todas las otras, sino en la relación abierta y franca que emerge de una conversación en la que están implicados todos los componentes de la universidad y que hace que la misma idea de educación liberal sea un constructo abierto a las modificaciones (McIntyre, 2013, 356-357). En esa conversación entre todos los componentes de una universidad y, por ende, entre las disciplinas, se hace posible unir los fines de los estudiantes y de la universidad en una unidad superior a través de una conversación entre diferentes voces. O, en palabras del filósofo inglés, la

búsqueda del conocimiento no es una carrera en la que los competidores se disputan el primer puesto, ni siquiera es un debate o un simposio, es una conversación. Y la virtud peculiar de la universidad (en calidad de espacio de diversos estudios) es demostrarlo en ese sentido, en el que cada estudio aparece como una voz cuyo tono no es tiránico ni retumbante, sino humilde y afable. Una conversación no necesita un director, no sigue un rumbo determinado de antemano, no nos preguntamos para qué «sirve» y no juzgamos su excelencia teniendo en cuenta su conclusión; no tiene conclusión, sino que siempre queda para otro día. No se impone su integración, sino que surge de la calidad de las voces que tienen la palabra, y su valor está en los recuerdos que va dejando en la mente de quienes participan de ella (Oakeshott, 2009b, 137).

Pero una vez Oakeshott ha considerado lo que significa comprender una universidad como una conversación, entonces, percibe que esa misma relación dialógica se modifica cuando se pasa al terreno de la política (Plotica, 2015, 123). Ahí, ni se puede aspirar a la sabiduría que brota del autoconocimiento ni es posible suspender el tiempo de las necesidades. Entonces, la meta se convierte en lograr una victoria que tenga una traducción en la vida práctica (Fuller, 2003, 44). Sin embargo, esta última apreciación que Oakeshott destina a la política constituye en Alasdair MacIntyre la esencia de la universidad y se erige como una metáfora con sentido propio.

\section{CUARTA METÁFORA: EL AGÔN}

MacIntyre comprende el diálogo no a la manera amable de Oakeshott y, desde luego, de una forma mucho más drástica que la de Hutchins cuando escribía que la finalidad de una discusión "no es el acuerdo, sino la comunicación" (Hutchins, 1959, 43). En efecto, MacIntyre comprende la universidad como un agôn. Serán 
necesarias unas breves aproximaciones al universo semántico de este concepto para entender mejor la metáfora macinteriana.

\subsection{Breve aproximación al concepto de "agôn"}

La idea del "agôn" ha permeado toda la cultura hasta la modernidad, cuando adquiere una particular relevancia con los trabajos de Nietzsche (Hatab, 2002), aunque, no obstante, está presente en las interpretaciones de la teoría política de Hannah Arendt (Robert-Miller, 2002) o, ya en un nivel intrafiducial, en el Martin Buber después de la Shoah (Forman-Barzilai, 2003). No obstante, la época en la que el "agôn" se hizo el centro de la vida social fue la Grecia clásica. Allí, "the word òyóv is used to indicate all kinds of contests» (Daqing, 2010, 6805) hasta el punto de que termina convirtiéndose en el espíritu de una época: impregna los juegos olímpicos, la política, la retórica, la filosofía, la educación (Hawhee, 2002). La palabra ha pervivido hasta hoy en el concepto de "agonía" como el sufrimiento extremo posterior a algún tiempo de batalla espiritual (op. cit., 6805) y está muy presente en algunas investigaciones farmacológicas (Brzozowski et al., 1997). Pero también ha sobrevivido en el ámbito de la FE gracias a las propuestas de Alasdair MacIntyre. En efecto, el pensamiento del escocés se puede leer como un agonismo encarnado en la historia de la filosofía cuyo desarrollo se ha realizado en las universidades (Cowlin, 1994).

\subsection{Alasdair MacIntyre y la concepción agonística de la universidad}

El pensamiento macinteriano sobre la universidad ha de comprenderse como la confluencia de dos conceptos que sirven para articular gran parte de su filosofía. Primeramente, la idea de "comunidad", que consistiría en una concepción moral compartida por todos sus miembros, concretada en una definición clara y diferenciada de las virtudes (MacIntyre, 2013, 182). Esto permite que los nuevos miembros de la comunidad puedan aprender esas virtudes, pero, sobre todo, permite que los individuos puedan comprender y hacer inteligibles sus vidas a sus ojos y a los de todos (MacIntyre, 2013, 258-259). Después, aparece el concepto de "tradición". Viene a ser el segmento de intersección entre la identidad comunitaria del individuo y la dimensión histórica de la comunidad (MacIntyre, 2013, 185-186). La complejidad acaece cuando los conceptos de virtud que manejaba la comunidad, y que transmitía a los recién llegados, comenzaron a interpretarse de distintas maneras, generando así una fuerte confusión sobre qué era cada virtud (Macintyre, 2013, 171). El punto de inflexión aquí fue Aristóteles. Porque consiguió condensar toda la tradición de pensamientos enfrentados anteriores (MacIntyre, 1994, 47-97) y articuló una doctrina que fue reinterpretada por otras tradiciones posteriores tanto para asimilar y profundizar en sus argumentos como para confrontarlos abiertamente. Lo que MacIntyre (1992) caracteriza como la tradición aristotélica, la enciclopédica y la genealógica. Aquí nace el «agôn» macinteriano. 
Como se puede observar, hay una dimensión del concepto de "tradición" en MacIntyre que opera de un modo netamente intelectual (MacIntyre, 2013, 271, 274275; 1994, 333-336) hasta el punto de que él mismo habla de «tradiciones intelectuales». ¿Pero qué es lo que hacen estas tradiciones? Sustancialmente, profundizar en los conceptos que han heredado de los autores y libros que les preceden, de tal modo que puedan articular nuevas interpretaciones que configuren una moral coherente para las personas que viven inmersas en la comunidad que se rige por esa tradición intelectual (MacIntyre, 1994, 333-336; MacIntyre, 2013, 191). Y aquí, en el momento en que estas tradiciones intelectuales se esfuerzan por profundizar en ellas mismas (MacIntyre, 1994, 333) y se enfrentan a las tradiciones rivales (MacIntyre, 1994, 31), aparece la universidad como un "agôn" (MacIntyre, 1992, 274). Porque estas tradiciones intelectuales se han mantenido vivas a través de las universidades y el discurso científico (Cowlin, 1994) y porque cada sistema universitario -casi cada universidad, diríamos- encarna cada una de esas tradiciones (MacIntyre, 1992, 274). ¿Pero cómo operaría el «agôn» en un contexto universitario?

Su dinámica experimentaría un doble movimiento. Por una parte, un movimiento ad intra (MacIntyre, 1994, 25-27, 333; Harris, 2012, 241). En él se situaría esa penetración en los conceptos a la luz de la propia tradición que ya sugerimos. Pero entonces, cuando se cree que se ha conseguido comprender un concepto tal como es, se produce un movimiento ad extra en donde esa interpretación se enfrenta a las tradiciones rivales (MacIntyre, 1994, 31). Entonces, las tradiciones que han salido derrotadas se retiran a su núcleo para volver a profundizar en la interpretación del concepto que no ha logrado salir vencedor y, así, permitir un desarrollo progresivo de la propia tradición a la luz de los sucesivos enfrentamientos. El «agôn» opera como el escenario que esclarece las interpretaciones más válidas que pueden permitir avanzar la moral y la política a través del discurso filosófico.

Una vez realizada esta descripción es fácil componer el resto de los elementos de la teoría educativa. Los profesores habrían de pertenecer a la tradición intelectual no solo a nivel cognoscitivo, porque comprendieran los principales nudos argumentativos, sino existencial, porque encarnarían las implicaciones morales de la tradición (MacIntyre, 1992, 284; Harris, 2012, 238-239). En este escenario, los estudiantes no acudirían a la universidad «solo» para la adquisición de un título que les facilite su posterior inserción laboral, sino, sin perjuicio de que esto fuera así en las sociedades modernas, para ser introducidos intelectual y moralmente en la tradición que encarna la universidad donde estudian (MacIntyre, 1992, 284 y ss.; MacIntyre, 1994, 379; MacIntyre, 2012, 274-275). Uno de los modos en el que los estudiantes son introducidos son los libros. Estos son centrales en el pensamiento de MacIntyre porque recogen la doctrina de una tradición intelectual, pero, sobre todo, porque se da una apropiación existencial de su contenido. No obstante, MacIntyre difiere aquí de la teoría educativa de Hutchins y Adler (MacIntyre, 1992, 281). Ellos proponían un recorrido cronológico, lineal, que MacIntyre no podía articular en una comprensión de bloques enfrentados. Precisamente por ello, los libros debían agruparse según las propias tradiciones y leerse intentando comprender 
las transformaciones conceptuales que se producían tanto en el interior de cada tradición como en el enfrentamiento con las demás (MacIntyre, 1992, 282). Por lo que el espíritu agonístico se encarna, en suma, también en pedagogía dentro del pensamiento de MacIntyre.

Pero, llegado un punto, MacIntyre comprendió la dificultad de aplicar una FE como la suya a la realidad actual de las universidades y los estudiantes. Entonces, articuló un programa de estudios que, más que buscar el duelo entre tradiciones, proponía una comprensión histórica de las grandes áreas que conforman la realidad con el fin de que cada estudiante adquiriera una visión de la realidad dotada de sentido y pudieran hacerse preguntas que tocaran el corazón de su existencia (MacIntyre, 2006). Pero, más que el contenido de esa propuesta de MacIntyre, se abre una pregunta que afecta a todas las metáforas anteriores. ¿Tiene sentido cualquiera de las teorías educativas que subyacen para la universidad de hoy?

\section{6. Últimas PALABRAS: A MODO DE CONCLUSIÓN}

El objetivo de este artículo ha sido sistematizar las diversas interpretaciones sobre la idea humanística de la universidad a través de las metáforas que condensaban sus principales elementos. Para ello, se han presentado el marco contextual de cada una de ellas, los fundamentos educativos que subrayan y las consecuencias que se derivan. Consecuencias, ya decimos, que aportan líneas de pensamiento relevantes para la universidad como idea, pero también para el cuerpo docente y estudiantil que las habita hoy día, con una serie de implicaciones pedagógicas que conviene detenerse a pensar. Justo a este ejercicio queremos dedicar estas últimas palabras, en la medida y con la profundidad que permite el espacio limitado del cierre de un artículo, con el fin de articular una propuesta de universidad humanista que armoniza lo más valioso de la tradición con el mejor porvenir.

La primera metáfora concebía a la universidad como una mónada cerrada en sí misma y es evidente que entraría en crisis en un momento en que tanto a universidades más localistas como 'grandes' se les demandara algo más. De esta crítica lógica se deduce que el futuro de las universidades pasa, necesariamente, por un diálogo con los diversos agentes sociales. O, dicho más específicamente, que también ha de entenderse como un espacio público y creativo del modo en que Dewey pensaba que las democracias son creativas: que los objetos del pensamiento pueden ser descubiertos y re-descubiertos continuamente en virtud de cada generación. Y es esta idea de apertura creativa la que se ofrece como clave hermenéutica de todo el resto de metáforas que presentamos. Porque en los Great Books convive esa tensión que busca, en el pasado, las respuestas a los desafíos del presente - ¿se puede entender de otro modo el renacimiento aristotélico que se condensa en la educación del carácter y el aprendizaje-servicio?-. Porque la conversación que se abría entre los libros y los lectores se amplía a todos los lenguajes que componen la sinfonía de la universidad, donde la palabra verdad resuena en diferentes tonos. Y esas voces, que mantienen posturas distantes, deben golpearse 
entre sí y con las demás para permitir discernir cuál de todas enfrenta esos desafíos de un modo más sabio. Si esta dinámica uniforme que presentamos, si esta hermenéutica sintética es cierta, la pregunta que queda por hacerse es la siguiente: ¿qué elementos de las metáforas anteriores pueden contribuir a conducir a las universidades actuales a una fidelidad mayor a sus orígenes en una nueva síntesis creativa abierta a los desafíos del momento?

Parece evidente que la primera idea de una universidad humanista pasa por la noción de "tradición". En efecto, todos los estudios que ofertan las universidades deberían ser capaces - a través de la función docente del profesorado, a veces no valorada suficientemente- de mostrar una historia del pensamiento humano que permita a los estudiantes conocer los orígenes y desarrollos de las ideas esenciales que configuran los problemas culturales, políticos, económicos y sociales del momento actual. Esto debería hacerse con un contacto más directo con los textos originales, pero presididos, más que por la búsqueda de una cierta erudición, por el desarrollo de un pensamiento crítico que abra nuevos horizontes. Es evidente, y esto hay que reconocerlo también, que la universidad actual está saturada de disciplinas cada vez más específicas, que originan planes de estudio muy heterogéneos y diversos. Pero esto no hace sino subrayar la necesidad, todavía más, de incardinar un currículum nuclear en detrimento de asignaturas demasiado periféricas y accesorias, especialmente en los títulos universitarios básicos. Ahora bien, esta idea habría de realizarse atendiendo a todas las expresiones del pensamiento humano, no únicamente textual, lo que lleva al estudio de una historia de las ideas abierta a todas las vías del conocimiento. Claro que hablamos de los principales nudos metafísicos y teológicos, pero también de los nuevos lenguajes en auge que se hacen imprescindibles para comprender el mundo actual: el matemático, biológico e informático y el conocimiento de las otras culturas. Es evidente, en suma, que todo pasa por pensar qué significa la «unidad del conocimiento" y su traducción curricular en una era de diversidad de lenguajes, traducidos en numerosas especializaciones.

La segunda idea tiene que ver con la dimensión teleológica de la universidad. Para este punto parece necesario alejarse de las comprensiones sobre la «verdad» de Newman, Hutchins, Adler y Oakeshott porque obviaron que los frutos de la búsqueda de la verdad no son solo cognoscitivos, sino performativos en un sentido amplio. Porque transforman al individuo que comprende algún matiz nuevo de la verdad, pero -acaso precisamente por ello- buscan transformar también a todas las personas que no pueden conocer por motivos económicos, sociales o políticos. Esto supone matizar la idea de que las disciplinas deban estar al servicio de la economía, la política o la industria militar, para subrayar que están al servicio de la persona y, especialmente, de las más necesitadas. En definitiva, la necesaria apertura de la universidad a la sociedad, que acaso encuentre su más alto grado de descentramiento en los márgenes de las sociedades.

Además, la búsqueda de la verdad solo puede hacerse en una comunidad donde profesores y estudiantes mantienen relaciones educativas de intercambio recíproco. Esta sería la tercera nota que es necesario mantener y reformular. Los 
profesores habrían de relacionarse entre sí no solo por el intercambio de conocimientos, lo cual es más enriquecedor cuanto más alejadas están sus disciplinas, sino también por el de los métodos de enseñanza y aprendizaje que usan y por las relaciones humanas, con los propios profesores y con los estudiantes, que configuran su sentido de pertenencia e identidad en la comunidad. Estas relaciones deben ampliarse a los estudiantes. Porque el modo de afrontar las situaciones existenciales por nuestros estudiantes, con características muy diversas, que trabajan o cuidan de sus familias mientras estudian, son lecciones fundamentales que mantienen vivo el corazón de la enseñanza. Y así, del atributo cardinal que preside una relación educativa como esta, acaso la reciprocidad, se irradian sus notas fundamentales al discurso científico y a la sociedad como en un crescendo que ayuda a ubicar mejor el papel de las universidades hoy.

En definitiva, parece que la línea de desarrollo de la interpretación de una universidad humanista en el siglo XXI al hilo de estas metáforas pasa por comprender dos categorías en todos los elementos posibles que una teoría educativa permite: la categoría de "relación» y la de «unidad". Ya hemos hablado de muchas de las relaciones que habrían de pensarse de nuevo. Por las pretensiones del estudio y los límites del manuscrito, no hemos podido analizar la relación de los profesores y los estudiantes con las disciplinas, por ejemplo, y de las distintas ramas de conocimiento entre sí. Pero sí que parece que todas han de pensarse en vistas a una «unidad» que se genere en la diversidad. Un conjunto de disciplinas, profesores y estudiantes que, a través de las múltiples relaciones que son capaces de generar entre sí, en común-unidad, buscan una verdad capaz de conquistar una humanidad nueva.

\section{REFERENCIAS BIBLIOGRÁFICAS}

Adler, M. J. (1977) Philosopher at large: an intellectual autobiography, 1902-1973. New York, Scribner Paper Fiction.

Adler, M. J. (1982) The Paideia Proposal. An educational manifesto. New York, MacMillan Publishing Company.

ADler, M. J. (1986) A Guidebook to Learning for the lifelong pursuit of the wisdom. New York, MacMillan Publishing Company.

ADler, M. J. (1988) Reforming education. The opening of American mind. New York, MacMillan Publishing Company.

Amilburu, M. ${ }^{a}$ G. (1997) Aprendiendo a ser bumanos. Una antropología de la educación. Pamplona, EUNSA.

BotTone, A. (2010) The Philosophical Habit of Mind. Bucharest, Zeta Books.

Bruce, B. C. (2011) John Dewey's Great Debates Reconstructed. Charlotte, NC., Information Age Publishing.

BRzOzOwsKi, A. M. et al. (1997) Molecular basis of agonism and antagonism in the oestrogen receptor. Nature, 389, 753-758.

Chaddock, K. E. y CoOKE, A. J. (2015) Endurance Testing: Histories of Liberal Education in U.S. Higher Education, en PAulsen, M. B. (ed.) Higher Education: Handbook of Theory and Research. New York, Springer International Publishing, 187-244. 
Chantler, A. (2016) The Ivory Tower revisited. Discourse: Studies in the Cultural Politics of Education, 37 (2), 215-229.

ClegG, S. (2008) Academic identities under threat? British Educational Research Journal, 34 (3), 329-345.

Cowlin, M. (1994) Alasdair MacIntyre, Religion and the University. The New Criterion, 12 (6), 1-9.

Culler, A. D. (1958) The Imperial Intellect. New Haven, Yale University Press.

Curren, R. (2007) Philosophy and the Necessity of Public Education. Philosophy Now, 63, 24-27.

Curren, R. (2017) Philosophy of Education: its current trajectory and challenges, en PETERS, M. A. (ed.) Encyclopedia of Educational Philosophy and Theory. Singapore, Springer, 1863-1866.

Dzuback, M. A. (1990) Hutchins, Adler and the University of Chicago: a critical juncture. American Journal of Education, 99 (1), 57-76.

Engel, S. M. (2007) Political Education in/as the Practice of freedom: a Paradoxical Defence from the perspective of Michael Oakeshott. Journal of Philosophy of Education, 3, 325-349.

EtzKowitz, H. et al. (2000) The future of the university and the university of the future: evolution of ivory tower to entrepreneurial paradigm. Research Policy, 29 (2), 313-330. doi: 10.1016/s0048-7333(99)00069-4

Forman-Barzilai, D. (2003) Agonism in Faith: Buber's Eternal Thou after the Holocaust. Modern Judaism, 23 (2), 156-179.

Franco, P. (2004) Oakeshott. An introduction. Great Britain, St. Edmundsbury Press.

Frowe, I. (2007) 'The politics of faith and the politics of scepticism': Michael Oakeshott, education and extremism. British Journal of Educational Studies, 55 (3), 264-285.

Fuller, T. (2003) The Idea of the University in Newman, Oakeshott, and Strauss. Academic Questions, 37-53.

García BARÓ, M. (2014) El peligroso momento actual de la universidad. Sal Terrae, 102 (1195), 939-952.

GARY, K. (2006) Leisure, freedom and liberal education. Educational Theory, 56 (2), 121-136.

GARY, K. (2017) Neoliberal Education for Work Versus Liberal Education for Leisure. Studies in Philosophy and Education, 36 (1), 83-94.

Glucksberg, S. y Keysar, B. (1993) How metaphor work, en Ortony, A. (ed.). Metaphor and Tought. New York, Cambridge University Press, 401-424.

Gomá, J. (2016) La misión de la universidad: formar profesionales y ciudadanos. El País, 15 de agosto.

Gracia, J. (2011) El intelectual melancólico. Un panfleto. Barcelona, Anagrama.

Green, Th. F. (1993) Learning without metaphor, en OrTONY, A. (ed.) Metaphor and Tought. New York, Cambridge University Press, 610-620.

HARRIS, S. (2012) The University's Uncommon Community. Journal of Philosophy of Education, 46 (2), 236-250.

Hatab, L. J. (2002) Prospects For A Democratic Agon: Why We Can Still Be Nietzscheans. The Journal of Nietzsche Studies, 24, 132-147.

Hawhee, D. (2002) Agonism and Aretê. Philosophy \& Rhetoric, 35 (3), 185-207.

HeldKe, L. (2005) Robert Maynard Hutchins, John Dewey and the Nature of the Liberal Arts. The Cresset. A review of literatura, the arts and public affairs, LXIX (2), 8-13. 
Himmelfarb, G. (1975) The Conservative Imagination: Michael Oakeshott. American Scholar, 44 (3), 405-420.

HochschiLD, J. P. (2003) The re-Imagined Aristotelianism of John Henry Newman. Modern Age, 45, 333-342.

Hutchins, R. M. (1936) The Higher Learning in America. New Haven, Yale University Press. Hutchins, R. M. (1959) La universidad de Utopía. Buenos Aires, EDubA.

Jover OlmedA, G. y Gozálvez, V. (2012) La universidad como espacio público: un análisis a partir de dos debates en torno al pragmatismo. Bordón, 64 (3), 39-52.

LACY, T. (2006) Making a democratic culture: the Great Book Idea, Mortimer J. Adler, and the twentieth-century America. Chicago, Ill., Loyola University Chicago.

LACY, T. (2008) Dreams of a culture: Revising the Origins of the Great Books Idea, 1869-1921. Society for Historians of the Gilded Age \& Pregressive Era, 7 (4), 397-441.

LACY, T. (2010) The Lovejovian Roots of Adler's Philosophy of History: Authority, Democracy, Irony, and Paradox in Britannica's "Great Books of the Western World». Journal of the History of Ideas, 71 (1), 113-137.

LACY, T. (2013) The Dream of a democratic culture. Mortimer J. Adler and the Great Books Idea. New York, Palgrave MacMillan.

LaKoff, G. y Johnson, M. (1980) We lived by metaphors. Chicago \& London, Chicago University Press.

LeE, M. J. (2012) The Conservative Canon and Its Uses. Rhetoric and Public Affairs, 15 (1), $1-39$.

Llovet, J. (2011) Adiós a las universidades. El eclipse de las Humanidades. Barcelona, Galaxia Gutenberg.

MacInTYRe, A. (1992) Tres visiones rivales de la ética. Madrid, Rialp.

MacInTYRe, A. (1994) Justicia y Racionalidad. Barcelona, EIUNSA.

MacInTYre, A. (2001) Catholic. Universities. Dangers, Hopes, Choices, en Sullivan, R. E. (ed.) Higher Learning and Catholic Traditions. Indiana, University of Notre Dame Press.

MacIntyre, A. (2006) The End of Education. The Fragmentation of American University. Commonweal. A Review of Religion, Politics and Culture, 133, 10-14.

MacInTYre, A. (2009) The very idea of a university: Aristotle, Newman and us. British Journal of Educational Studies, 57 (4), 347.

Macintyre, A. (2012) Dios, filosofía, universidades. Historia selectiva de la tradición filosófica católica. Granada, Nuevo Inicio.

MacInTYRe, A. (2013) Tras la virtud. Barcelona, Austral.

Martin, J. R. (1994) Changing the Educational Landscape: Philosophy, Women, and Curriulum. New York, Routledge.

Martínez, M. A.; Sauleda, N. y Huber, G. L. (2001) Metaphors as blueprints of thinking about teaching and learning. Teaching and Teacher Education, 17, 965-977.

McGrath, F. (1951) Newman's University. Idea and Reality. London, Longmans Green \& Co.

MCINTYRE, K. B. (2013) Liberal education and the teleological question; or Why should a dentist read Chaucer? Journal of Philosophy of Education, 47 (3), 341-363.

Newman, J. H. (1997) Ensayo sobre el desarrollo de la doctrina cristiana. Salamanca, Bibliotheca Oecumenica Salmanticensis.

Newman, J. H. (2011) Discursos sobre el fin y la naturaleza de la educación universitaria. Navarra, EUNSA.

OAkeshotт, M. (2002) Experience and its modes. New York, Cambridge University Press. 
OAKeshotT, M. (2003) On buman conduct. New York, Oxford University Press.

OAKeSHOTT, M. (2009a) La actitud conservadora. Madrid, Sequitur.

OAKeshotT, M. (2009b) La voz del aprendizaje liberal. Madrid, Katz.

OAKESHOTT, M. (2013) Sobre la historia y otros ensayos. Madrid, Katz.

OAKeshott, M. (2017) Ser conservador y otros ensayos escépticos. Madrid, Alianza editorial.

PÉrez díAz, V. (2010) Universidad, ciudadanos y nómadas. Oviedo: Ediciones Nobel.

Peters, R. S. (1968) Michael Oakeshott's philosophy of education, en KING, P. y PAREKH, B.

C. (eds.) Politics and experience. Cambridge, Cambridge University Press, 43-63.

Petrie, H. G. y Oshlag, R. S. (1993) Metaphor and learning, en Andrew Ortony, A. (ed.) Metaphor and Tought. New York, Cambridge University Press, 579-609.

Plotica, L. K. (2015) Michael Oakeshott and the Conversation of Modern Political Thought. New York, Sunny Press.

Scott Johnston, J. (2011) The Dewey-Hutchins debate: a dispute over moral teleology. Educational Theory, 61 (1), 1-16.

Searle, J. (1993) Metaphor, en Ortony, Andrew (ed.) Metaphor and Tought. New York, Cambridge University Press, 83-111.

SHAPIN, S. (2012) The Ivory Tower: the history of a figure of speech and its cultural uses. BJHS, 45 (1), 1-27.

SHIN, A. Y. (2018) Outside of the Ivory Tower. Techniques in Hand \& Upper Extremity Surgery, 22 (2), 37-38.

SHRIMPTON, P. (2014) The 'Making of Men'. The Idea and reality of Newman's university in Oxford and Dublin. Leominster, Gracewing.

SIEGEL, H. (1981) How 'practical' should philosophy of education be? Educational Studies: a Journal of the American Educational Studies Association, 12 (2), 125-134.

Standish, P. (2003) The nature and purposes of education, en CuRren, Randall (ed.) A Companion of Philosophy of Education. United Kingdom, Blackwell Publishing, 221-231.

STEvens, A. H. (2001) The philosophy of general education and its contradictions: the influence of Hutchins. The Journal of General Education, 2001, 50 (3), 165-191.

Tillman, K. (2008) 'A Rhetoric in Conduct': The Gentleman of the University and the Gentleman of the Oratory. Newman Studies Journal, 5 (2), 6-25.

Tingley, E. (2002) Knowledge for the Sake of Knowledge. First Things, 119, 15-17.

TrIPP, S. (1992) Michael Oakeshott's Philosophy of Education and its Implications for Instructinal Design Theory and Practice. Consultado el 03 de febrero de 2018. https://files.eric. ed.gov/fulltext/ED353951.pdf.

Visser-WijnVEEN, G. J. et al. (2009) The relationship between academics' conceptions of knowledge, research and teaching -a metaphor study. Teaching in Higher Education, 14 (6), 673-686.

Weltman, B. (2002) Individualism versus socialism in American education: rereading Mortimer J. Adler and the Paideia Proposal. Educational Theory, 52 (1), 61-79.

Williams, K. (2007) Education and the voice of Michael Oakeshott. Thoverton, Exeter and Charlotesville, va, Imprint Academic.

WRIGHT, L. (1999) On the passivity of Newman's 'gentleman': negotiating incoherence. English Studies: A Journal of English Language and Literature, 3, 239.

ÚCAR, X. (2018) Metáforas de la intervención socioeducativa: implicaciones pedagógicas para la práctica. Revista Española de Pedagogía, 76 (270), 209-224. doi: 10.22550/ REP76-2-2018-01. 